Canadian

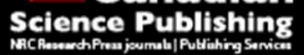

Canadian Journal of Physics

Revue canadienne de physique

\title{
Cosmology, Cosmic Dust, and Stellar Dimming
}

\begin{tabular}{|r|l|}
\hline Journal: & Canadian Journal of Physics \\
\hline Manuscript ID & cjp-2015-0732.R1 \\
\hline Manuscript Type: & Article \\
\hline Date Submitted by the Author: & 02-Mar-2016 \\
\hline Complete List of Authors: & Matthias, Chuck; Softext Publishing Inc. \\
\hline Keyword: & $\begin{array}{l}\text { cosmology, stellar dimming, cosmic dust, redshift model, stellar recession } \\
\text { model }\end{array}$ \\
\hline \multicolumn{2}{|l}{} \\
\hline
\end{tabular}

SCHOLARONE ${ }^{\text {M }}$

Manuscripts 


\section{Cosmology, cosmic dust, and stellar dimming}

Chuck. S. Matthias

Softext Publishing Inc.

Owen Sound, ON, N4K 4K5, CAN

csmphysics9@gmail.com

\section{Abstract:}

A cosmology model is derived, forming an expanding, curved, unbounded, and finite universe resembling a 3-dimensional hypersphere with positive curvature. Growth, curvature, and the expansion rate are forced by the initial conditions accompanying the emergence of space and time from the "big bang" singularity. The model generates several variables (past and present stellar distance, luminosity distance, time of emission, photon path, recession velocity, radiation temperature, universe circumference, and Hubble parameter) which are sole functions of redshift $z$ (readily measured by spectroscopic or photometric means). The model also presents two concepts which replace the need for cosmic inflation and dark energy.

Equations for luminosity distance $\mathrm{d}$ and Hubble parameter $\mathrm{H}$ compare extremely well with 28 values of BAO measured data over the redshift range $0.07<z<2.3$. A second data set, compiled by NASA, consists of 27000 SN1a supernovae measurements of luminosity distance and redshift $(0.001<z<10)$. Although the data are extremely scattered, within the scatter is a narrow, well-defined core whose distance $d^{*}$ exceeds the model value $d$ as redshift increases. The larger distance $\mathrm{d}^{*}$, resulting from a weaker than expected optical signal, is commonly explained as due to an acceleration of universe expansion. Alternatively, if the photons are assumed to be partially quenched by cosmic dust, in proportion to the distance travelled, the weakened core signal can be described by a radiative transfer equation. The resulting equation for $\mathrm{d}^{*}$, with an extinction coefficient of $0.000345 \mathrm{Mpc}^{-1}$ (or a photon mean free path of $2900 \mathrm{Mpc}$ ), fits the core data perfectly.

Keywords: cosmology, stellar dimming, cosmic dust, redshift model, stellar recession model

\section{PACS: 90, 95, 98}

\section{INTRODUCTION}

Cosmology can be defined as the description of the general structure of the universe from its origin, through the present, and into the future. Most cosmologies are built using Einstein's equations of general relativity (GR), in particular the Friedmann equations. The GR equations describe the distribution of energy and matter as a function of the curvature of space and time, and vice versa. The Friedmann equations describe the effects of radiation, pressure, gravity, dark energy, dark matter, ordinary matter, curvature, etc. upon the expanding universe in time 
and space and require the evaluation of several constants (up to 6) in order to optimize the fit of the equations to astronomical measurements.

GR equations are essential for analysis of strong gravitational fields, for rapid motion through space, and for the analysis of properties within space in general. The present study develops a heuristic non-GR model which describes the growth of space as a function of cosmic time $\mathrm{T}$. All clocks which are at rest with respect to local space, and which are remote from large masses or energy sources, measure the same cosmic time $\mathrm{T}$, even if space is expanding. To measure the growth of space, a relatively simple geometric analysis is adequate in which we can ignore the internal dynamics of mass and energy. Space behaves as an expanding fabric which contains countless billions of masses, of which stars are crucially important in transmitting information on the history of the universe. Stars are moved through space by nearby gravitational fields and move with space as space expands with cosmic time. Since we are interested only in the expansion of space, data will be gathered from deep space stars for which recession speeds exceed gravitationally-induced speeds. The accuracy of the model is established by a comparison of measurement and theory for (1) the luminosity distance $d$ and for (2) the Hubble parameter $\mathrm{H}$. These variables, and all others, will be expressed solely as a function of redshift $z$. In this paper, "space" and "universe" are synonymous, finite, 3dimensional, and exist only for an instant of time between a finite past and an uncertain future. Details of the model geometry will be suggested by the following 2 tenets which are a mix of philosophy, conjecture, and observation.

Firstly, the model adheres to the cosmological principle, i.e., that our position in the universe is not privileged, and that our observations of the physical universe (space and contents) on the large scale are no different from observations made at any other location. This implies that the universe has no centre, no edge, is unbounded, and is homogeneous and isotropic on the large scale. Any radiation generated within the universe will circle indefinitely unless intercepted (i.e., microwave background radiation, primordial neutrinos, stellar photons). This topology is difficult to visualize for a 3 dimensional universe, but not for 2 dimensions (surface of a balloon) or 1 dimension (circumference of a circle). If our universe is homogeneous and isotropic, stellar information from all space directions is equivalent. Only the distance is important, and so for descriptive purposes (Fig. 1), the 3 physical dimensions can be collapsed into a 1 dimensional circle of circumference $C n=2 \pi R n(n=n o w)$, wrapped around a second inaccessible dimension of radius $\mathrm{Rn}$, whose centre is not part of the physical universe. This model is a simplified description of our postulated universe, which would be in reality a 3 dimensional hypersphere, with positive curvature defined by the inaccessible $4^{\text {th }}$ dimension radius $\mathrm{Rn}$. It is also an example of symmetry, which is common in nature.

Secondly, observations of stellar redshift indicate that the universe is expanding. The redshift results from spatial circumferential stretching in the 1 dimensional model (see outer circle of Fig. 1), driven by spatial and temporal growth in the radial direction. These assumptions and observations are satisfied by a model in which both cosmic time $\mathrm{T}$, and space, (the latter containing fundamental constants, radiation, mass, 3 length dimensions, etc.) erupt instantaneously from a singularity in a void, popularly referred to as the "big bang". The singularity is located at the origin (Fig. 1), with time T (or equivalent distance R) being the radius. We further assume (1) that time and space are contiguous as they move out together radially through the void, occupying a thin temporal band at the radius $T$ (or $R$ ), and (2) that the radial movement through the void proceeds at the newly created constant speed of light ' $c$ ', giving $\mathrm{R}=\mathrm{c} T$. 
The circumferential growth of Fig. 1 models the 3 dimensional growth of the universe which contains a dynamic system of mass and energy. These dynamics affect the orbital movements of planets and stars within 3 dimensional space, but have no effect on the expansion of space itself, or on the $4^{\text {th }}$ dimension expansion of time. This leads to a simple set of equations which can be compared to measurements within the universe for verification. In summary, within the void, the radius of curvature of the 3-dimensional universe grows at the speed of light and GR does not apply; within the universe, GR controls mass-energy dynamics.

There are a few papers available favouring the $R=c T$ universe, developed from the Friedmann equations. One which was brought to my notice is by Melia et al. [1]. The $R=c T$ assumption in general constrains the Friedmann equations to describe a very specific universe having (1) no mass or energy, (2) a cosmological coefficient of zero, and (3) a spatial curvature index of negative 1. The Newtonian argument in their Appendix A argues that spatial curvature is zero. The present heuristic model is independent of universe mass and energy, has no cosmological constant, and has positive curvature. The best judge of models, as usual, will be the quality of comparison of data with theory.

The information pathway from an emitting star Se in the past at time Te is shown by the dashed line in Fig. 1 and is explained in Section 2. Fig. 1 is the space-time history of the universe.

In Section 2, the cosmology model is constructed from geometric principles. From it, we derive several stellar functions expressed analytically in terms of redshift $z$. Recent data are available for comparison to the variables $\mathrm{H}$ and $\mathrm{d}$. Since $\mathrm{z}$ is readily measured, a quantitative description of the past stellar cosmos (at cosmic time $\mathrm{T}$ ) is easily provided from the redshift. We shall also examine the stellar luminosity distance aberration (between model and measurement).

\section{DERIVATION OF EQUATIONS}

From Fig. 1, consider a case where light is emitted from star $\mathrm{Se}(\mathrm{e}=\mathrm{emitting})$ at time $\mathrm{Te}=$ $0.5 \mathrm{Tn}$ and moves toward earth Ee along arc xe. The position of Se in the hypersphere spacetime is defined by distances $\operatorname{Re}$ and $x e$, or $\operatorname{Re}$ and angle $\theta$ (we are choosing to keep $\theta$ positive to assure that distances $x e$ and $x n$, and velocities xe' and xn', are all positive). Note that angle $\theta$ is fixed for each star. With the universe expanding and the stellar photons moving at a finite speed c, the signal arrives at earth En at time Tn by moving circumferentially through the universe along the expanding arc which grows to the present length of $\mathrm{xn}$. At the same time, the universe (or circumference) has expanded radially through the void, carrying Se to a new location Sn. The locus of points of the photons travelling through space and time is given by the dashed line labelled ' $d s$ '. This path is related to the luminosity distance $d$ which will be discussed in Section 2c. These photons are the only means for measuring space-time properties of an image from the past (such as radial velocity, and distance). It will be shown later that this path is unique. Although all stars at radius $\mathrm{Re}$ are emitting at time Te, photons from those at larger (or smaller) $\theta$ will arrive at earth at later (or earlier) times than Tn. Other stars which contribute to En are located at the intersection of universe circles with the photon path 'ds', which extends back in time to the stelliferous period. The locus of all these intersections (also shown in Fig. 1) is the total source of the starlight arriving at En. The photon path curls indefinitely and more rapidly around the origin as Re and Te shrink to zero.

\section{2a. Basic Geometrical Equations}


Several equations follow from the geometry of Fig. 1. These are

$R n=c T n, \quad \operatorname{Re}=c T e, \quad x n=\operatorname{Rn} \theta, \quad x e=\operatorname{Re} \theta$, from which we find velocities

$\mathrm{Rn}^{\prime}=\mathrm{Re} \mathrm{e}^{\prime}=\mathrm{c}, \quad \mathrm{xn} \mathrm{n}^{\prime}=\mathrm{xe} \mathrm{e}^{\prime}=\mathrm{c} \theta=\mathrm{v}$ (recession velocity). From these, we can determine the Hubble relations

$x n^{\prime} / x n=1 / T n\left(=H n=R n^{\prime} / R n=c / R n\right), \quad x e^{\prime} / x e=1 / T e\left(=H e=R e^{\prime} / R e=c / R e\right)$.

In addition,

$\mathrm{Tn} / \mathrm{Te}=\mathrm{Rn} / \mathrm{Re}=\mathrm{xn} / \mathrm{xe}=\mathrm{He} / \mathrm{Hn}$

Perhaps the most interesting of these equations is the Hubble relation $\mathrm{H}$, expressed as $x^{\prime} / x=1 / T$, where $x^{\prime}$ and $x$ are measured at the same cosmic time $T . H$ is often stated casually as the ratio of stellar radial (or recession) velocity divided by stellar distance. For the star Se, the recession speed xe' ( $\left.=x n^{\prime}\right)$ can be calculated from a redshift measurement from the observatory at En, but xe is not directly measurable. To find the Hubble relation $\mathrm{He}$ at $\mathrm{Se}, \mathrm{xe}$ cannot be replaced by the luminosity distance $\mathrm{d}$. A model is required for either xe or for He. (It turns out that $\mathrm{He}$ can be found from Eqs. (6) if $\mathrm{Hn}$ is known, or from Eq. (13) if the luminosity distance $d$ is measured.)

From the above relations, we see that a given star has a constant recession velocity from the earth of $x^{\prime}=c \theta$ for all cosmic time $T$. This is to be expected since the scale size $R$ has a constant growth rate. (More generally, any two points in space, free of gravitational influence, have a constant recession velocity from each other). The present value of the Hubble parameter can be considered to be the limit of $H(T)$ as T approaches $\mathrm{Tn}$. Since $\mathrm{Hn}$ can only be determined by measurement, and since recent measured values differ by only a few percent, the value used in this paper is $70 \mathrm{~km} \mathrm{~s}^{-1} \mathrm{Mpc}^{-1}$. From Eqs. $1, \mathrm{Tn}=1 / \mathrm{Hn}$ which gives us $\mathrm{Tn}=14$ billion years. (These values are corroborated by their use in the stellar distance measurements of Section 3.) From these, the radius $\mathrm{Rn}$ of our hypersphere is 14 billion light years, and our universe size $\mathrm{Cn}$ (circumference) is 88 billion light years.

\section{2b. Stretching of Space}

Missing in the above equations is a relation between recession velocity, redshift, and time which is vital in interpreting stellar data. A paper by Bunn et al. [2] provides the final equation along the photon path, linking events at time Te to events at time Tn. Consider a photon leaving Se at time Te and moving toward Ee. It travels for a short distance $\underline{\mathrm{dx}}=\mathrm{c} \underline{\mathrm{dT}}$ toward an observer fixed in space at a distance $\underline{\mathrm{dx}}$ from Se (differentials are underlined to avoid confusion with $x$ and $d$ and other letters used separately). Because space is continually expanding, the observer and the star are drifting apart, causing a small relative velocity $\underline{\mathrm{dv}}$ between star and observer. From Eqs. (1), the relative velocity created by the expansion is given by the Hubble relation $x^{\prime}=x / T$ in which we replace $x^{\prime}$ with $\underline{d v}$ and $x$ with $\underline{d x}$ giving

$\underline{\mathrm{dv}}=\underline{\mathrm{dx}} / \mathrm{T}=\mathrm{c} \underline{\mathrm{dT}} / \mathrm{T}$ 
This relative velocity causes an infinitesimal Doppler redshift at the observer and is given accurately by the nonrelativistic formula

$\underline{\mathrm{d} \lambda} / \lambda=\underline{\mathrm{dv}} / \mathrm{c}=\underline{\mathrm{dT}} / \mathrm{T}$

By integrating all the infinitesimal redshifts from the star Se to the observer at En, Eqs. (3) become

$\ln (\lambda \mathrm{n} / \lambda \mathrm{e})=\mathrm{v} / \mathrm{c}=\ln (\mathrm{Tn} / \mathrm{Te})$

This equation is valid for any redshift since there was no violation of special relativity at any point on the trajectory. The total Doppler shift of light from the star as it recedes from earth is $(\lambda n-\lambda e) / \lambda e=z$, and the total recession velocity is $v\left(=x e^{\prime}=x n^{\prime}=c \theta\right)$. Since $\lambda n / \lambda e=$ $1+z$, then, from Eqs. (4) and (1)

$\mathrm{v} / \mathrm{c}=\ln (1+\mathrm{z})=\theta$, and

$\lambda \mathrm{n} / \lambda \mathrm{e}=\mathrm{Tn} / \mathrm{Te}=1+\mathrm{z}=\mathrm{He} / \mathrm{Hn}=\mathrm{Rn} / \mathrm{Re}=\mathrm{xn} / \mathrm{xe}=\exp (\mathrm{v} / \mathrm{c})=\exp (\theta)$

Since Se is one of the points on the photon path (Fig. 1), then the locus of all the possible locations of Se will determine the photon path. The polar coordinate form of the path is

$\operatorname{Re}=\operatorname{Rn} \exp (-\theta)$

The Cartesian coordinate form is

$x=\operatorname{Re} \cos (\theta)$, and $y=\operatorname{Re} \sin (\theta)$, or

$x=R n \exp (-\theta) \cos (\theta)$, and $y=R n \exp (-\theta) \sin (\theta)$

These coordinates define a unique photon path between Se and En since $\theta$ and $\operatorname{Re}$ are unique functions of redshift $z$. Furthermore, all stellar properties discussed here are unique functions of $z$.

This Doppler analysis assumes that the thermal random velocity of the radiation source (atoms in a star) and gravitationally-induced motion of the star are negligible compared to the space expansion-induced velocity of the star relative to earth En. The atoms are referred to as the last point of contact for the photon. Prior to this Doppler analysis, we had a simple relation between time $T$ and the universe scale factor $\mathrm{R}$ (Eqs. 1). Using the Doppler scheme, we are able to link T, R, the redshift of $\lambda$, and the stretching of space in a causative way (Eqs. (6)). Using the Hubble relations from Eqs. 1 and velocity $v$ from Eqs. 5, the variables xe and $\mathrm{xn}$ can be expressed as

$x e=c \operatorname{Te} \ln (1+z)=c \operatorname{Tn} \ln (1+z) /(1+z), x n=c \operatorname{Tn} \ln (1+z)$ 
In the early stages of expansion ( $\mathrm{Te} / \mathrm{Tn}<<1$ ), the universe is tiny, radiation dominated, and growing rapidly. From Eqs. (4) and (5), we see that the turning angle $\theta=\ln (\mathrm{Tn} / \mathrm{Te})$ is enormous, and decreasing rapidly since $\mathrm{d} \theta / \mathrm{dTe}=-1 / \mathrm{Te}$. For every $2 \pi$ change of $\theta$, the photons circle the universe, in all directions, maintaining a well-mixed radiation medium. As it cools, particles form, creating a charged plasma that blocks the radiation. The pulsating plasma creates sound waves which circle the universe at near light speed and continue the mixing. At the same time, the universe is expanding and stretching out inhomogeneities on the large scale. These processes eliminate the need to postulate an enormous "inflation" event to homogenize the early universe. Furthermore, the baryon acoustic oscillations (BAO) may create density fluctuations of sufficient magnitude to assist in gravitational clumping for the genesis of stars. These fluctuations continue until cosmic time $\mathrm{Te}=12.7$ million years (to be shown shortly) when the universe temperature falls below 3000 degrees $\mathrm{K}$ (Kelvin) and the plasma becomes neutral. The equilibrium radiation distribution is released and the relatively quiescent hydrogen density variations gradually succumb to the pull of gravity, continuing the long slow process of accretion and star formation. The density clumping by BAO may obviate the need for "dark matter" to assist in star formation.

Radiation is the key factor which links the universe from beginning to end. In the early universe, when the temperature $\boldsymbol{T}$ was large, radiation was in a black-body equilibrium with ionized matter. The plasma temperature $\boldsymbol{T}$ and the wavelength $\lambda \mathrm{m}$ (at the peak of the energy density spectrum) are related in Wien's Law as

$\lambda \mathrm{m} \boldsymbol{T}=\mathrm{b}$ (a constant), from which we find

$\operatorname{Te} / \mathrm{Tn}=\lambda \mathrm{n} / \lambda \mathrm{e}=1+\mathrm{z}$

As the universe cooled and became neutral, the radiation was no longer trapped and the spectral distribution travelled unimpeded and unchanging to the future, where it was discovered in the 1960's as the cosmic microwave background radiation (CMBR). When the spectrum was measured and analysed, the peak wavelength $\lambda \mathrm{m}$ was strongly Doppler redshifted relative to its last contact with the equilibrium plasma at $T \mathbf{T}=3000$ degrees $\mathrm{K}$ such that the present value of $z$ equaled 1100. Using Eqs. (10), the present radiation temperature is $\boldsymbol{T n}=2.73$ degrees $\mathrm{K}$. From Eqs. (5), the turning angle $\theta$ of the radiation source is 400 degrees, implying that the CMBR radiation arriving now at our solar system began its journey when the universe was small $(\mathrm{Te} / \mathrm{Tn}=.00091=\mathrm{Re} / \mathrm{Rn})$ and circled the universe completely, prior to its arrival. Evaluating Te, the model finds the time of neutralization to be about 12.7 million years after the "big bang".

This time period is much larger than more common estimates of 380,000 years which arise from the $\Lambda$ CDM cosmology model, preferred among most cosmologists. $\Lambda$ CDM describes a "big bang" followed by decelerating growth because of gravitational forces, followed by an indefinitely growing acceleration caused by hypothetical "dark energy". Such deceleration and acceleration are disallowed by the assumptions of the present model, as described in Section 1. Furthermore, $\triangle \mathrm{CDM}$ is a complex model, with the simplest form requiring the evaluation of 6 parameters to make a best fit to data. The model of this paper has only 1 undetermined parameter $(\mathrm{Hn})$, commonly measured to be $70 \mathrm{~km} \mathrm{~s}^{-1} \mathrm{Mpc}^{-1}$, and verified as such by the model-data agreement in Section 3. 


\section{2c. Luminosity Distance d}

In Fig. 1, the only remaining variable to evaluate is the luminosity distance $d$ associated with the photon path between Se and En. The luminosity distance is an important variable since it can be measured with confidence by photometric techniques applied to standard candles (such as Cepheids or supernovae). It is part of the relation between the power output of the star (known for standard supernovae) and the measured energy flux received on earth (see Eq. (14)). If the modelled and measured distances are in agreement, then we can have confidence in the other stellar variables derived above.

The photon path (Eq. (7a)) is a classic logarithmic spiral which has a length given by

$\mathrm{ds}=2^{1 / 2} \operatorname{Rn}(1-\exp (-\theta))=2^{1 / 2} \operatorname{cTn} z /(1+\mathrm{z})$

As mentioned at the beginning of Section 2, the photon path has 2 orthogonal components (radial and circumferential). The circumferential component describes the curved motion of the photons travelling through the universe from Se (at time Te) to En (at time Tn); the radial component describes the lateral displacement of the expanding universe through the void. Each component grows at the speed of light c for a time period $\mathrm{Tn}-\mathrm{Te}$ and they are consequently equal. The luminosity distance ' $d$ ' is the circumferential component given by

$\mathrm{d}=\mathrm{c}(\operatorname{Tn}-\operatorname{Te})=\mathrm{cTn}(1-\mathrm{Te} / \operatorname{Tn})=\mathrm{cTn}(1-1 /(1+\mathrm{z}))=\mathrm{cTn} z /(1+\mathrm{z})$

from which it is evident that $d s^{2}=d^{2}+d^{2}$. Note that the space-time radius $c T n=c / H n=$ $300,000 \mathrm{~km} \mathrm{~s}^{-1} / 70 \mathrm{~km} \mathrm{~s}^{-1} \mathrm{Mpc}^{-1}=4286 \mathrm{Mpc}$ (= 14 billion light years) which is used in Eq. (11b) to plot luminosity distance as a function of $z$. Eq. (11b) implies that any source in the past, during which photons had free and unobstructed movement, is detectable if the signal is strong and the detector is sensitive. Some of the original photons arrive at En, even from the most remote source, despite the continual growth of the pathway between source and receptor. This is possible because the remote source now was not remote 14 billion years ago. Equation (11b) also tells us that the most distant source possible in the limit of large $z$ (or early time) is presently at $4286 \mathrm{Mpc}$. Since the first stars are believed to have formed at about $\mathrm{T}=250$ million years, the earliest stellar signal would have travelled a maximum distance of about $4200 \mathrm{Mpc}$. Furthermore, this path is our only information pathway from the past.

\section{2d. Equation Summary}

The equations are displayed in three ways. The modelled variables are plotted nondimensionally vs redshift to show the behaviour of all the variables on a single graph. Secondly, they are plotted dimensionally along with measured data to assess their validity. Thirdly, they are printed in Table 1 for redshifts ranging from .001 to 100000 .

The following non-dimensional equations are plotted in Fig. 2:

$\mathrm{v} / \mathrm{c}=\theta=\mathrm{xn} /(\mathrm{cTn})=\ln (1+\mathrm{z})$ 


$$
\begin{aligned}
& x e /(c T n)=\ln (1+z) /(1+z) \\
& d /(c T n)=z /(1+z) \\
& \operatorname{Te} / \operatorname{Tn}=\operatorname{Re} / \operatorname{Rn}=1 /(1+z) \\
& \mathrm{He} / \mathrm{Hn}=1+z
\end{aligned}
$$

These equations describe several properties for every star in the universe whose light arrives at planet earth at the present time ( $T n=14$ billion years). Each star is described uniquely by a redshift $z$. All stars with the same $z$ will have common properties as described by the equations of Section 2.

The first row of equations describes $v$ (recession speed), $\theta$ (turning angle), and $\mathrm{xn}$ (present distance of star being observed). They grow as identical monotonic functions, rapidly with small $z$, and more slowly with large $z$. The velocity $v$ may exceed the speed of light $c$ without violating rules of special relativity since the recession is "of space" rather than "through space". The distance $x e$ to the emitting star rises to a maximum at $z=1.718$ and then falls continually. This implies that Se and Ee were closer to each other in the early universe (large $z$ ) when the universe itself was very small. The luminosity distance $d$ to the stellar image lies between xe and $\mathrm{xn}$ and asymptotes to a maximum value of cTn. The emission time Te recedes rapidly to zero for large $z$. The Hubble relation $\mathrm{He}$ is the inverse of $\mathrm{Te}$, increases linearly with large $\mathrm{z}$, and will be shown to be in excellent agreement with measured data.

A number of other expansion properties are listed in Table 1 as a function of redshift. Several lengths are written as "B light years" or billion light years.

\section{LUMINOSITY DISTANCE AND HUBBLE PARAMETER: DATA AND MODEL}

These models are assessed using 2 data sets. The first is a compilation of 28 measured Hubble parameters $\mathrm{H}(\mathrm{z})$ or $\mathrm{He}$ (Farooq et al. [3]) arising from baryon acoustic oscillation (BAO) data. The second data set (Steer et al. [4]) is a much larger NASA database of redshiftindependent extragalactic distances (NED-D). NED contains nearly 80000 distance measurements, but of these, only 27000 distances have redshift measurements. These are luminosity distances, determined by measuring the brightness of the signal and comparing it with the computed signal from a standard candle model.

The modelled Hubble parameter He appears in Eqs. (12). It is plotted in Fig. 3 and is in excellent agreement with the Farooq et al. [3] He data. This is the largest compilation of $\mathrm{He}$ data at present, ranging over redshifts $.07<z<2.3$.

Since Eqs. (12) are sole functions of $z$, they are functions of each other. From Eqs. (12), we find

$\mathrm{d}=\mathrm{cz} / \mathrm{He}$

which allows us to convert Farooq et al. [3] He data into luminosity distance data d. Measured and modelled distances are plotted in megaparsecs vs redshift $z$ in Fig. 4 and are also in excellent agreement. Of particular importance is the fact (1) that BAO data do not depend on stellar brightness, and (2) there is no deviation from the model that would suggest an acceleration of the universe. Consequently, the assumption of uniform expansion is corroborated by the BAO data. 
The NASA stellar database is publically available on the web. The present paper uses all the data for which both luminosity distance and redshift are available (about 27000 measurements). All NED-D data with redshift exceeding 1.55 are gamma ray bursts (GRB); most of the remainder are SN1a supernovae. Data and model (d from Eqs (12)) are plotted in Fig. 5. The data are plotted as small translucent points so as to make a density pattern on the graph. The model distance is the solid red line and is an excellent fit to the dense core data (heavy black line) for values of $z$ less than 0.1 . For larger $z$, the model falls below the core and its projection (dashed red line). The good fit at low $z$ implies that the analytical form of $d$ is locally accurate, and that the universe lifetime $\mathrm{Tn}$ and the Hubble parameter $\mathrm{Hn}$ are also correct $\left(\mathrm{Hn}=70 \mathrm{~km} \mathrm{~s}^{-1} \mathrm{Mpc}^{-1}=1 / \mathrm{Tn}\right)$. More generally, we can say that $\mathrm{Tn}=\mathrm{d}(1+\mathrm{z}) /(\mathrm{cz})$, at least in the limit of small $z$.

The data distribution of Fig. 5 displays abundant visual information. It is important to recall that the data come from 3 dimensions (all sky sectors). The most important display is the narrow dense dark line (or core) which defines a line of maximum probability for the distance-redshift path. The second prominent feature, suggestive of significant photon extinction, is the extensive faint haze above the core. A third weaker feature is the faint narrow haze slightly below the core.

The dense core appears to be the primary path for the photons (having minimal scatter, and clearly visible for $0.01<z<1.0$ ). It is a remarkably tight formation, implying that the universe is quite homogeneous since the data have come from many sky sectors. More specifically, it is homogeneous with respect to universe expansion, which causes stars at a given distance $d$ to have the same recession velocity (or redshift $z$ ). The narrow core broadening with distance and its displacement upwards suggest that the photons have traversed a moderately uniform cosmic dust field and are absorbed in proportion to the distance travelled. The weakened signal causes the observed distance to exceed the model distance $d$ (solid red line).

Secondly, the faint haze at greater distances results from photons which have travelled from sky sectors containing additional patches of non-uniform debris. The universe is not homogeneous with respect to cosmic dust and debris.

Thirdly, the haze below the core implies that these star distances are closer than expected, which cannot result from photon extinction. The distribution has a well-defined lower edge and so does not appear to be due to experimental error. This downward displacement could result from supernovae which are brighter than the norm, causing them to appear closer than predicted by the standard nuclear fusion model luminosity.

To clarify the scatter, the data were re-plotted as small opaque points in Fig. 6 . These data are much more visible in the high redshift range $1<z<10$, compared to those of Fig. 5 . Despite the paucity of data in this range, the central trend seems to follow the core projection (dashed red line), curving slowly away from the anticipated true stellar distance d (solid red line). The Fig. 6 data more clearly define the clean lower edge $(0.01<z<1)$ which is parallel to and just below the luminosity model $d$.

The physics of standard candles is quite accurate, judging from the excellent agreement of the distance model Eq. (11b) with the measured core data for low redshift. Absorption or scattering of core radiation can be estimated in the mean by using a simplified radiative transfer equation. If the total power production at any time from the star can be calculated to be $Q$ watts, and if there is no absorption of the signal as it travels to earth En, the net radiative energy flux $q$ that we receive over a distance $d$ is

$q d^{2}=Q / 4 \pi \quad(d=$ luminosity distance $)$ 
If there are losses on route, the reduced flux $q^{*}$ received is given by

$\mathrm{q}^{*} \mathrm{~d}^{2}=\exp (-\mathrm{m} \mathrm{d}) \mathrm{Q} / 4 \pi$

where $\mathrm{m}$ is a mean absorption coefficient (unknown) for the radiation, and its inverse $I_{\mathrm{m}}=\mathrm{m}^{-1}$ is the photon mean free path. Furthermore, if there are losses, we have unknowingly misused Eq. (14) which will give us instead the product $q^{*} d^{* 2}$, where $d^{*}$ will be an overestimate of the luminosity distance (displayed as the dense core of Fig. 5). Hence we can rewrite the above equations to be

$\mathrm{Q} / 4 \pi=\mathrm{q}^{*} \mathrm{~d}^{* 2}=\exp (\mathrm{m} d) \mathrm{q}^{*} \mathrm{~d}^{2}$

from which we find

$\mathrm{d}^{*} / \mathrm{d}=\exp (\mathrm{m} \mathrm{d} / 2)$

where $\mathrm{d}^{*}$ is the apparent luminosity distance of points along the high density core.

To calculate $\mathrm{m}$, we choose a redshift $\mathrm{z}=1$ for which $\mathrm{d}^{*}$ median value is about $3100 \mathrm{Mpc}$ (Fig. 5). Assuming the model (Eq. (11b)) to be accurate and unbiased, the 'true distance' $d$ is about $2143 \mathrm{Mpc}$. From Eq. (17), we find $\mathrm{m}=0.000345 \mathrm{Mpc}^{-1}$, and $I_{m}=\mathrm{m}^{-1}=2900 \mathrm{Mpc}$. Using the same equation, we can calculate the mean apparent luminosity distance $\mathrm{d}^{*}$ as a function of $z$. The dashed red line is the modelled path of $d^{*}$ for $z>1$. This explanation for the apparent augmentation of stellar recession negates the popular hypothesis of a "dark energy" which is accelerating the universe expansion, thereby weakening the stellar signal. Accelerated expansion could displace the core, but would not cause the random data scatter.

The fractional loss of photons due to quenching is given by

$\left(q-q^{*}\right) / q=1-\exp (-m d)$

which for a travel distance of $1000 \mathrm{Mpc}$ is about $29 \%$.

A final compelling argument that the data are erring to the high side is that the maximum travel time for the earliest stellar light is about 13.75 billion years, which converts to a maximum distance of about $4200 \mathrm{Mpc}$ (from Section 2c). Corresponding supernovae observations at $4200 \mathrm{Mpc}$ (the core data or apparent distance $\mathrm{d}^{*}$ ) have a measured redshift of 1.7. Data beyond this redshift are sparse and scattered, but most exceed the limit of $4200 \mathrm{Mpc}$ and indicate measurements which are impossibly large, as do the projected core data (dashed red line).

In summary, the model for luminosity distance $d$ appears to be accurate. The departure of the NED data from the model, as $z$ increases past 0.1 , can be explained very simply by light absorption, avoiding the unnecessary complexity of an accelerating universe expansion supported with large uncertainties by a multi-parameter curve fitting process. The BAO data are independent of measured light intensities, yielding a Hubble parameter and stellar distance (Figs. 3 and 4 ) that are in agreement with the model over the entire redshift range of $0.07<z<$ 2.3. This strongly supports the $R=c T$ hypothesis.

\section{CONCLUSIONS}

Equations are derived which describe the space-time behaviour of our universe from its origin at $\mathrm{T}=0$ to the present. In early cosmic time, the universe was small and photons circled it rapidly and randomly, as did baryon acoustic oscillations, maintaining a thermodynamic equilibrium, negating the need for "inflation", and possibly the need for "dark matter. A third postulate, "dark energy", is also negated since the supernovae stellar dimming is readily 
explained by cosmic dust. (Dark matter and dark energy are so far undetectable, even though they are purported to make up $95 \%$ of the universe energy content.)

The concept and the equations are simple and show excellent agreement with BAO Hubble parameter data. The agreement is such that there is no need to seek corrections in either the data or the model. We also have good agreement with the large NED-D data set of which we used 27000 records of luminosity distance and redshift. When the model is modified to include photon quenching by cosmic dust, the agreement with the core data is excellent. All stellar variables have been described as analytical functions of redshift alone. The simplicity and accuracy of the models suggest that the universe is well behaved, finite, unbounded, and in a steady expansion. Even signals from the most remote stars, receding at superluminal speeds, can be received and interpreted.

Occam, Newton, Leibniz, and others concluded that of two reasonable explanations, the one requiring the fewest assumptions is most likely to be correct. This principle favours dimming by cosmic dust over dimming by expansion acceleration.

The universe is closed, with positive curvature, but will continue to expand forever since the universe mass has no effect on the growth of space.

This cosmology structure gives a lucid description of the universe topology, growth, stellar recession, and photon pathways not from stars "far out in an infinite universe", but from stars "deep within a finite universe".

\section{ACKNOWLEDGEMENT}

This research has made use of the NASA/IPAC Extragalactic Database (NED), which is operated by JPL, Caltech, under contract with NASA.

\section{REFERENCES}

1. F. Melia, and A. S. H. Shevchuk. Mon. Not. R. Astron. Soc. 419, 2579 (2012).

2. E. F. Bunn, and D. W. Hogg. Am. J. Phys. 77, 688 (2009).

3. O. Farooq, and B. Ratra. Astrophys. J. Lett. 766, 1 (2013).

4. I. Steer, and B. F. Madore. NED-D: A Master List of Redshift-Independent Extragalactic Distances. http://ned.ipac.caltech.edu/. (2014)

\section{FIGURE CAPTIONS}

Fig. 1 A schematic of space-time from the "big bang" (origin) to the present physical universe (outer circle). The radius is the cosmic time $T$, extending to the present era $T n(n=n o w)$. The equivalent distance is $\mathrm{Rn}=\mathrm{cTn}$ (c=speed of light). Light emitted from a star Se (e=emitting) at time Te arrives at earth En at time Tn along the trajectory ds. The distances xe and xn are the stellar distances from earth at times Te and Tn. The dotted and dashed line from the origin is the photon trajectory from the origin to En.

Fig. 2 Stellar recession properties expressed non-dimensionally as a function of redshift $z$. The solid black line describes recession velocity v/c, present stellar distance $\mathrm{xn} / \mathrm{Rn}$, and angle $\theta$. The others are Hubble parameter He/Hn (solid red), luminosity distance d/Rn of stellar image (black dash), distance xe/Rn to emitting star from Ee (solid blue), and time Te/Tn at emission (red dash). 
Canadian Journal of Physics

Page 12 of 19

12

Fig. 3 Hubble parameter in km/s/Mpc vs. redshift $\mathrm{z}$ for model He (solid line) and measurements (circles).

Fig. 4 Luminosity distance in Apc vs. redshift $z$ for model $d$ (solid line) and measurements (circles).

Fig. 5 Luminosity distance in Apc vs. redshift $z$ for model d (solid red line), model $d^{*}$ (broken red line), and data from gamma ray bursts and supernovae (27000 tiny semi-opaque black dots).

Fig. 6 (same as Fig. 5, except that data points are opaque)

TABLE Table 1 has its own caption.

https://mc06.manuscriptcentral.com/cjp-pubs 


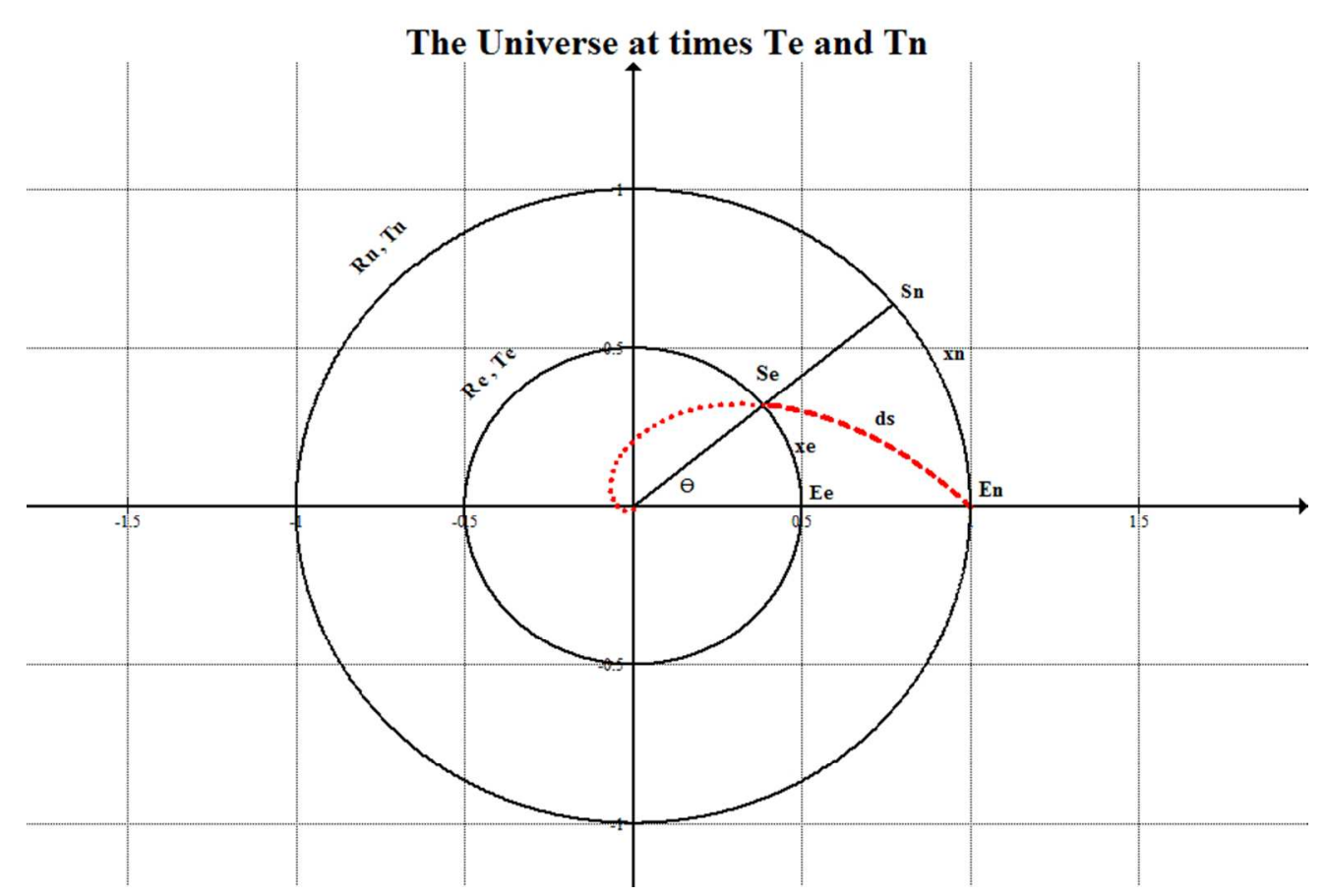

A schematic of space-time from the "big bang" (origin) to the present physical universe (outer circle). The radius is the cosmic time $T$, extending to the present era $T n$ ( $n=n o w)$. The equivalent distance is $R n=c T n$ ( $c=$ speed of light). Light emitted from a star Se (e=emitting) at time Te arrives at earth En at time Tn along the trajectory ds. The distances $x e$ and $x n$ are the stellar distances from earth at times Te and Tn. The dotted and dashed line from the origin is the photon trajectory from the origin to En.

$331 \times 224 \mathrm{~mm}(72 \times 72$ DPI $)$ 
Stellar Recession Properties

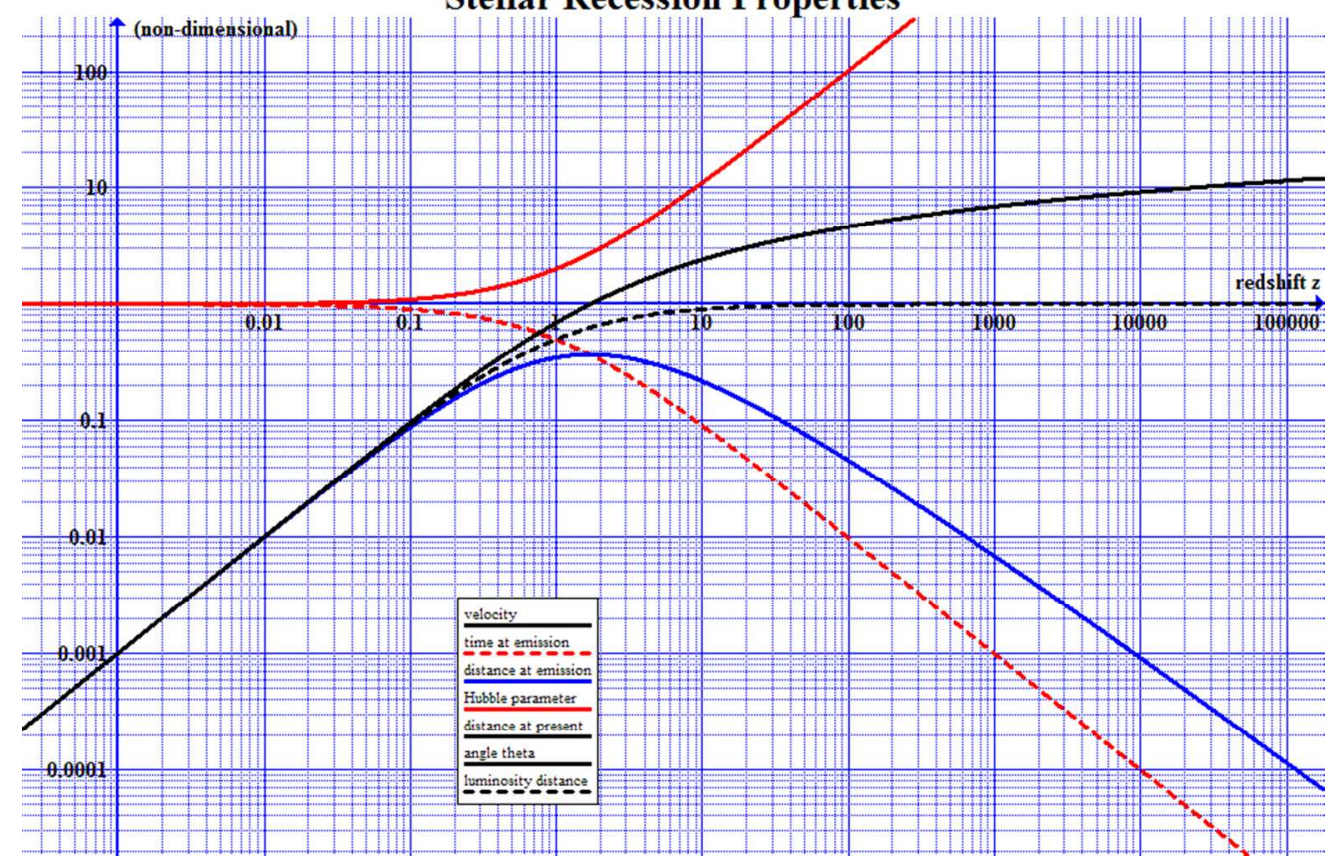

Stellar recession properties expressed non-dimensionally as a function of redshift $z$. The solid black line describes recession velocity $\mathrm{v} / \mathrm{c}$, present stellar distance $\mathrm{xn} / \mathrm{Rn}$, and angle $\theta$. The others are Hubble parameter $\mathrm{He} / \mathrm{Hn}$ (solid red), luminosity distance d/Rn of stellar image (black dash), distance xe/Rn to emitting star from Ee (solid blue), and time Te/Tn at emission (red dash).

$331 \times 224 \mathrm{~mm}(72 \times 72 \mathrm{DPI})$ 


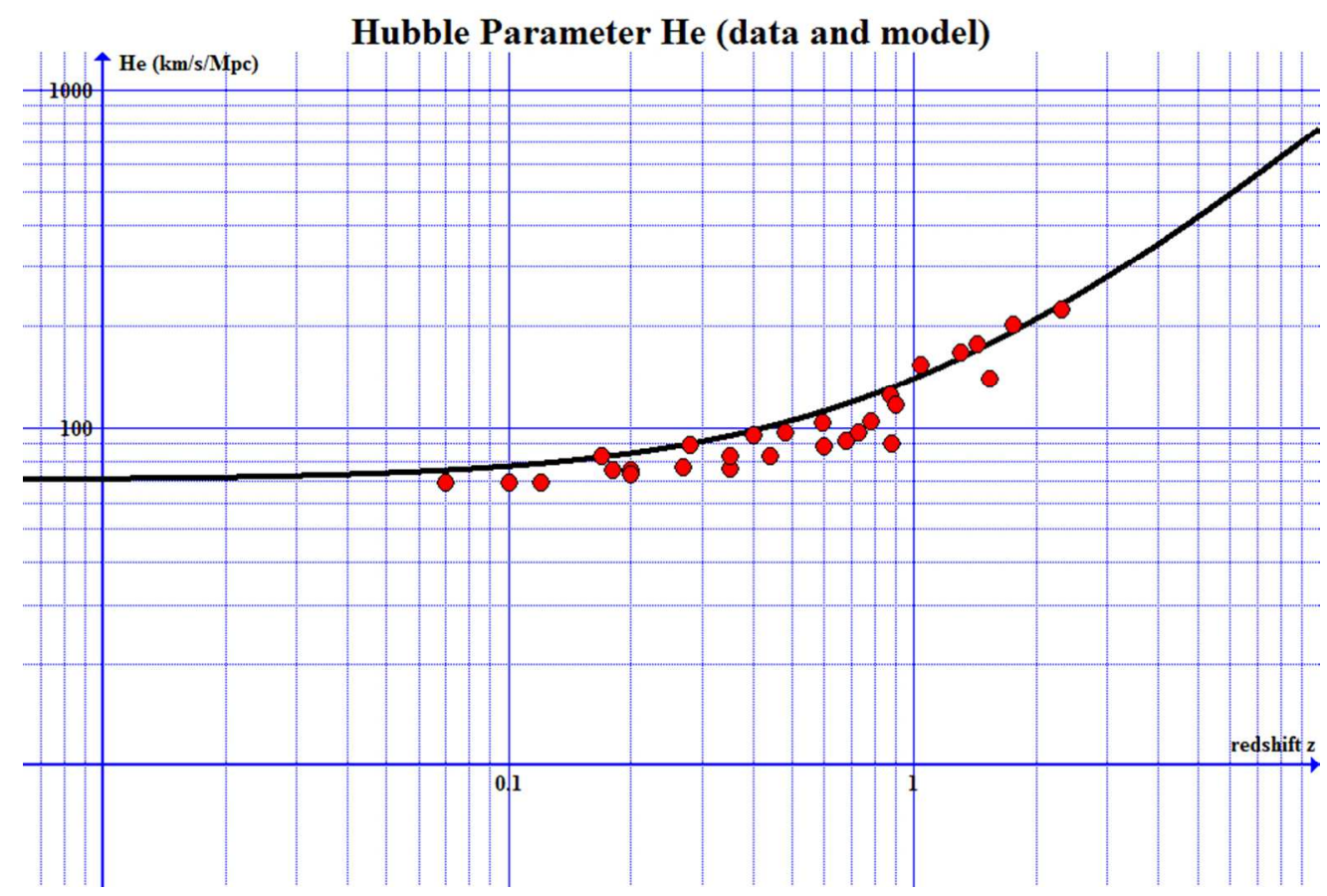

Hubble parameter in km/s/Mpc vs. redshift $\mathrm{z}$ for model He (solid line) and measurements (circles). $331 \times 224 \mathrm{~mm}(72 \times 72$ DPI $)$ 


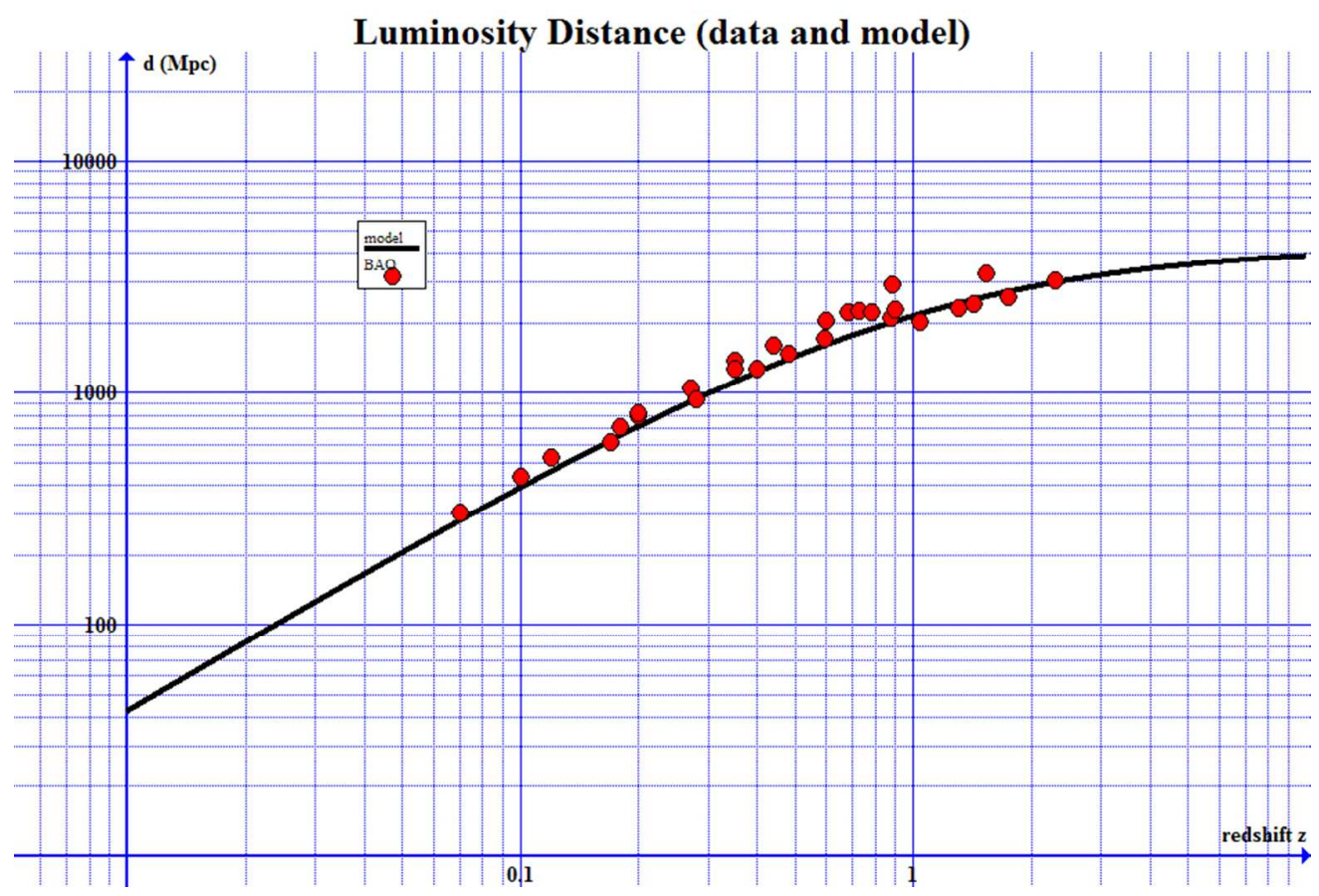

Luminosity distance in Mpc vs. redshift $z$ for model $d$ (solid line) and measurements (circles). $331 \times 224 \mathrm{~mm}(72 \times 72$ DPI $)$ 


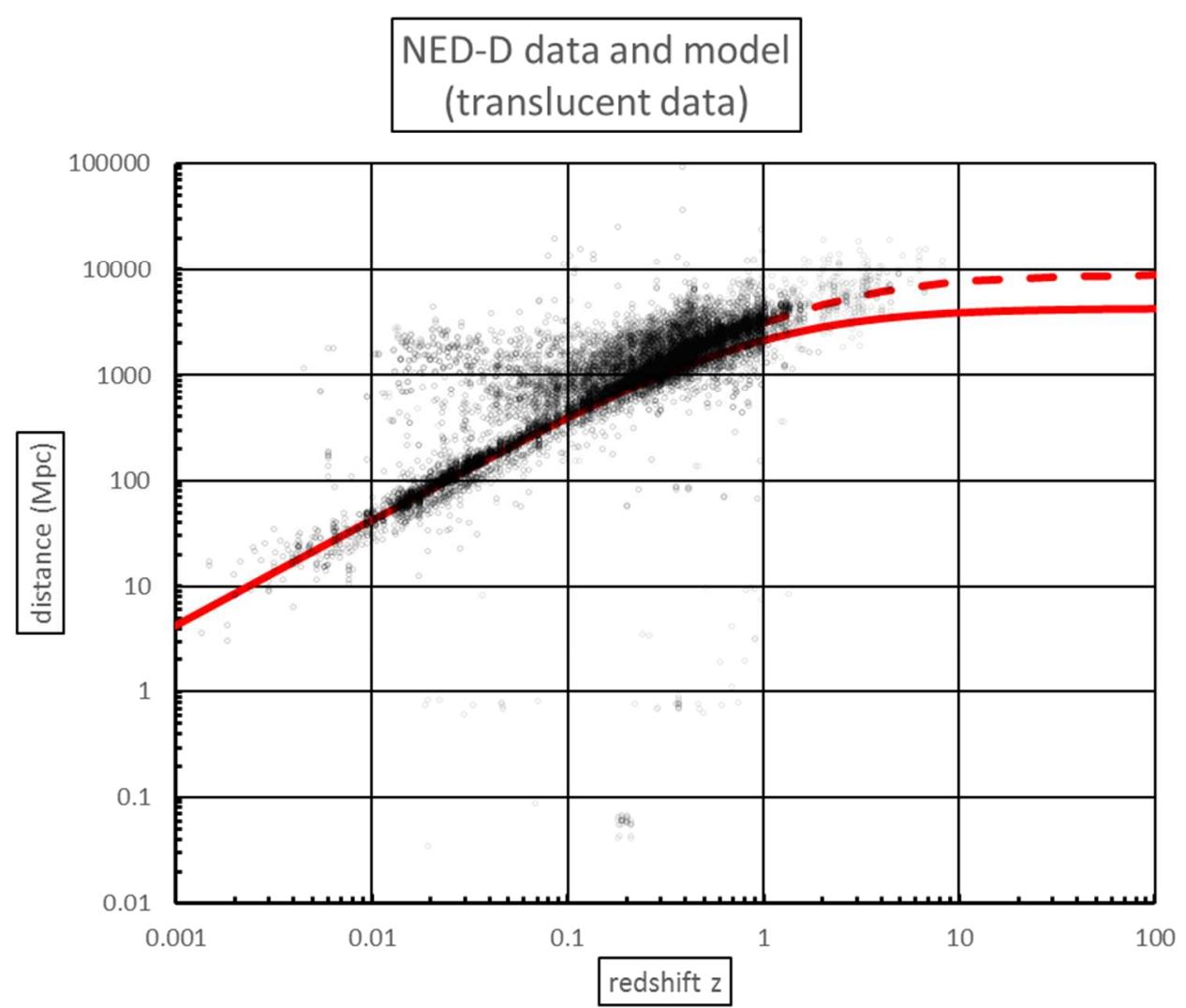

Luminosity distance in Mpc vs. redshift $\mathrm{z}$ for model $\mathrm{d}$ (solid red line), model $\mathrm{d}^{*}$ (broken red line), and data from gamma ray bursts and supernovae (27000 tiny semi-opaque black dots). 


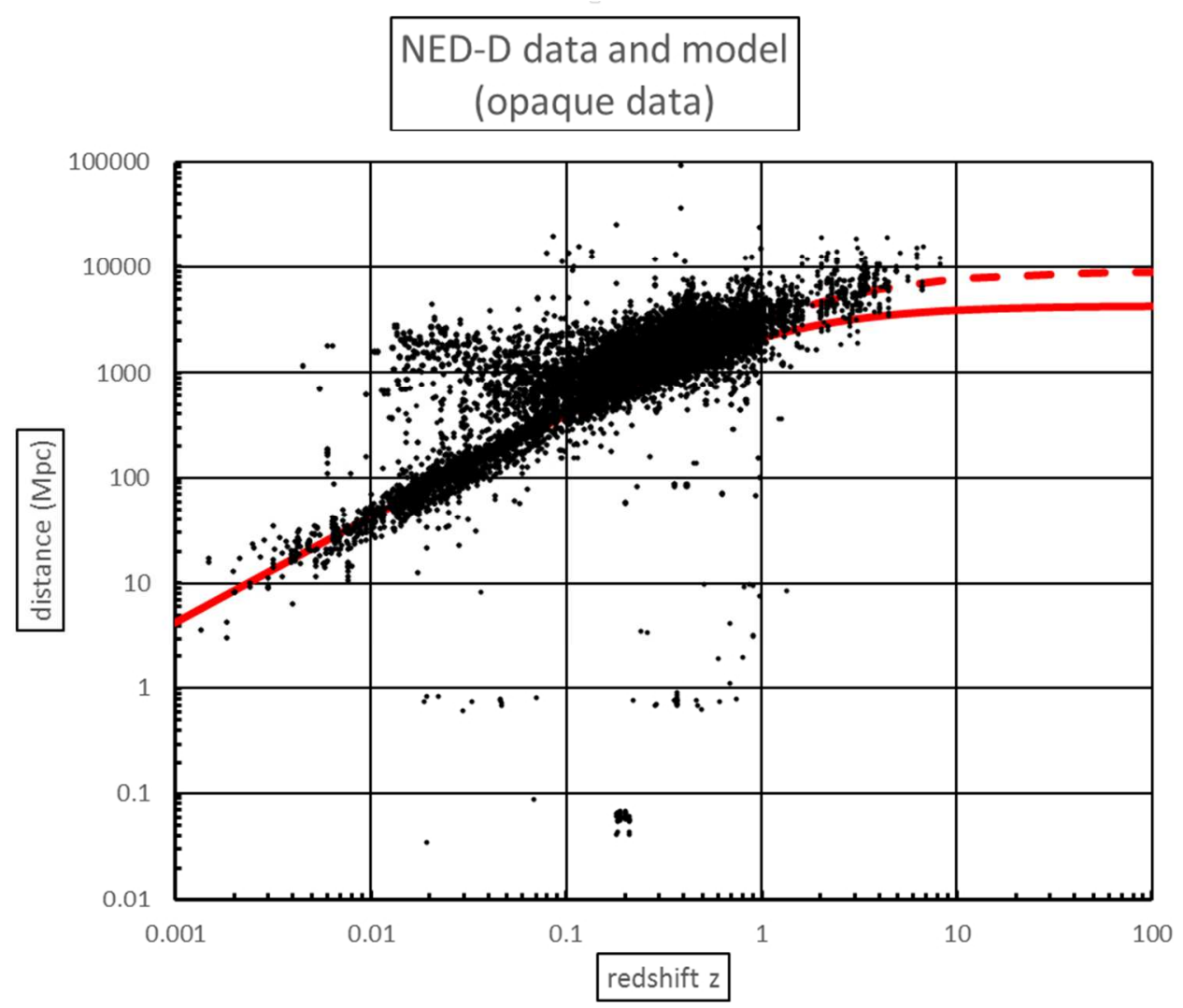

(same as Fig. 5, except that data points are opaque) 
Table 1 Stellar and Universe properties as a function of redshift

\begin{tabular}{|c|c|c|c|c|c|}
\hline$\stackrel{<}{\text { redshift }}$ & $\mathrm{Re} / \mathrm{R}_{\mathrm{II}}$ & $\begin{array}{c}\theta \\
\text { degrees }\end{array}$ & $\mathrm{Te} / \mathrm{T}$ ı & $v / c$ & $\begin{array}{c}\text { Re } \\
\text { B light yr }\end{array}$ \\
\hline 0.001 & 9.99E-01 & 0.06 & 9.99E-01 & $1.00 \mathrm{E}-03$ & $1.40 \mathrm{E}+01$ \\
\hline 0.003 & 9.97E-01 & 0.17 & 9.97E-01 & $3.00 \mathrm{E}-03$ & $1.39 \mathrm{E}+01$ \\
\hline 0.01 & 9.90E-01 & 0.57 & $9.90 \mathrm{E}-01$ & $9.95 \mathrm{E}-03$ & \\
\hline 0.03 & 9.71E-01 & 1.69 & $9.71 \mathrm{E}-01$ & $2.96 \mathrm{E}-02$ & $1.36 \mathrm{E}+01$ \\
\hline 0.1 & $9.09 \mathrm{E}-\mathrm{O}$ & 16 & $9.09 \mathrm{E}-01$ & $9.53 \mathrm{E}-02$ & $7 E+01$ \\
\hline 0.3 & 7.69E-01 & 15.03 & 7.69E-01 & 2.62E-01 & $1.07 \mathrm{E}+01$ \\
\hline 1 & 5.00E-01 & 39.72 & $5.00 \mathrm{E}-01$ & $6.93 \mathrm{E}-01$ & $6.99 E+00$ \\
\hline 3 & $2.50 \mathrm{E}-01$ & 79.43 & $2.50 \mathrm{E}-01$ & $1.39 \mathrm{E}+00$ & $3.49 E+00$ \\
\hline 10 & $9.09 \mathrm{E}-02$ & 137.40 & $9.09 \mathrm{E}-02$ & $2.40 \mathrm{E}+00$ & $1.27 \mathrm{E}+00$ \\
\hline 30 & $3.23 \mathrm{E}-02$ & 196.77 & $3.23 \mathrm{E}-02$ & $3.43 E+00$ & 4.51E-01 \\
\hline 100 & $9.90 \mathrm{E}-03$ & 264.45 & $9.90 \mathrm{E}-03$ & $4.62 E+00$ & $1.38 \mathrm{E}-01$ \\
\hline 300 & 3.32E-03 & 327.02 & $3.32 \mathrm{E}-03$ & $5.71 \mathrm{E}+00$ & 4.64E-02 \\
\hline 1000 & $9.99 \mathrm{E}-04$ & 395.87 & 9.99E-04 & $6.91 \mathrm{E}+00$ & $1.40 \mathrm{E}-02$ \\
\hline 3000 & 3.33E-04 & 458.78 & $3.33 \mathrm{E}-04$ & $8.01 \mathrm{E}+00$ & 4.66E-03 \\
\hline 10000 & $1.00 \mathrm{E}-04$ & 527.76 & $1.00 \mathrm{E}-04$ & $9.21 \mathrm{E}+00$ & $1.40 \mathrm{E}-03$ \\
\hline 30000 & 3.33E-05 & 590.70 & $3.33 \mathrm{E}-05$ & $1.03 \mathrm{E}+01$ & 4.66E-04 \\
\hline 100000 & $1.00 \mathrm{E}-05$ & 659.69 & $1.00 \mathrm{E}-05$ & $1.15 \mathrm{E}+01$ & $1.40 \mathrm{E}-04$ \\
\hline
\end{tabular}

\begin{tabular}{|c|c|c|c|c|c|}
\hline$z$ & $\mathrm{Te}$ & xe & $\mathrm{xn}$ & d & Te \\
\hline edshift & $B$ years & B light yr & B light yr & B light yr & $\operatorname{deg} \mathrm{K}$ \\
\hline 0.001 & $1.40 \mathrm{E}+01$ & 1.39E-02 & $1.40 \mathrm{E}-02$ & $1.40 \mathrm{E}-02$ & $2.73 \mathrm{E}+00$ \\
\hline 0.003 & $1.39 \mathrm{E}+01$ & 4.17E-02 & 4.18E-02 & $4.18 \mathrm{E}-02$ & $2.74 \mathrm{E}+00$ \\
\hline 0.01 & $1.38 \mathrm{E}+01$ & 1.38E-01 & $1.39 \mathrm{E}-01$ & $1.38 \mathrm{E}-01$ & $2.76 \mathrm{E}+00$ \\
\hline 0.03 & $1.36 \mathrm{E}+01$ & 4.01E-01 & 4.13E-01 & 4.07E-01 & $2.81 \mathrm{E}+00$ \\
\hline 0.1 & 1.27E+01 & $1.21 \mathrm{E}+00$ & $1.33 \mathrm{E}+00$ & 1. $27 \mathrm{E}+00$ & $3.00 \mathrm{E}+00$ \\
\hline 0.3 & $1.07 \mathrm{E}+01$ & $2.82 \mathrm{E}$ & $3.67 \mathrm{E}+00$ & $3.22 \mathrm{E}+00$ & $3.55 E+00$ \\
\hline 1 & $6.99 \mathrm{E}+00$ & $4.84 \mathrm{E}+00$ & $9.68 \mathrm{E}+00$ & $6.99 \mathrm{E}+00$ & $5.46 \mathrm{E}+00$ \\
\hline 3 & $3.49 \mathrm{E}+00$ & $4.84 \mathrm{E}+00$ & $1.94 \mathrm{E}+01$ & $1.05 \mathrm{E}+01$ & $1.09 \mathrm{E}+01$ \\
\hline 10 & $1.27 \mathrm{E}+00$ & $3.05 \mathrm{E}+00$ & $3.35 \mathrm{E}+01$ & $1.27 \mathrm{E}+01$ & $3.00 \mathrm{E}+01$ \\
\hline 30 & 4.51E-01 & $1.55 \mathrm{E}+00$ & $4.80 \mathrm{E}+01$ & $1.35 \mathrm{E}+01$ & $8.46 \mathrm{E}+01$ \\
\hline 100 & 1.38E-01 & $6.38 \mathrm{E}-01$ & $6.45 \mathrm{E}+01$ & $1.38 \mathrm{E}+01$ & $2.76 \mathrm{E}+02$ \\
\hline 300 & 4.64E-02 & $2.65 \mathrm{E}-01$ & $7.97 \mathrm{E}+01$ & $1.39 \mathrm{E}+01$ & $8.22 \mathrm{E}+02$ \\
\hline 1000 & $1.40 \mathrm{E}-02$ & 9.64E-02 & $9.65 \mathrm{E}+01$ & $1.40 \mathrm{E}+01$ & $2.73 \mathrm{E}+03$ \\
\hline 3000 & 4.66E-03 & $3.73 \mathrm{E}-02$ & $1.12 \mathrm{E}+02$ & $1.40 \mathrm{E}+01$ & $8.19 \mathrm{E}+03$ \\
\hline 10000 & $1.10 \mathrm{E}-03$ & 1.29E-02 & $1.29 \mathrm{E}+02$ & $1.10 \mathrm{E}+01$ & $2.73 \mathrm{E}+01$ \\
\hline 30000 & 4.66E-04 & $4.80 \mathrm{E}-03$ & $1.44 \mathrm{E}+02$ & $1.40 \mathrm{E}+01$ & $8.19 E+04$ \\
\hline 100000 & $1.40 \mathrm{E}-04$ & $1.61 \mathrm{E}-03$ & $1.61 \mathrm{E}+02$ & $1.40 \mathrm{E}+01$ & $2.73 E+05$ \\
\hline
\end{tabular}

